\title{
The metallicity gradient as a tracer of history and structure: the Magellanic Clouds and M33 galaxies
}

\begin{abstract}
M.-R. L. Cioni
University of Hertfordshire, Science and Technology Research Institute, Hatfield AL10 9AB, UK

e-mail: M.Cioni@herts.ac.uk

Received 24 March 2009 / Accepted 9 August 2009

ABSTRACT

Context. The stellar metallicity and its gradient place constraints on the formation and evolution of galaxies.

Aims. This is a study of the metallicity gradient of the LMC, SMC and M33 galaxies derived from their asymptotic giant branch (AGB) stars.

Methods. The $[\mathrm{Fe} / \mathrm{H}]$ abundance was derived from the ratio between $\mathrm{C}$ - and M-type AGB stars and its variation analysed as a function of galactocentric distance. Galaxy structure parameters were adopted from the literature.

Results. The metallicity of the LMC decreases linearly as $-0.047 \pm 0.003 \mathrm{dex} \mathrm{kpc}^{-1}$ out to $\sim 8 \mathrm{kpc}$ from the centre. In the $\mathrm{SMC}$, [Fe/H] has a constant value of $\sim-1.25 \pm 0.01 \mathrm{dex}$ up to $\sim 12 \mathrm{kpc}$. The gradient of the M33 disc, until $\sim 9 \mathrm{kpc}$, is $-0.078 \pm 0.003 \mathrm{dex} \mathrm{kpc}^{-1}$ while the outer disc/halo, out to $\sim 25 \mathrm{kpc}$, has $[\mathrm{Fe} / \mathrm{H}] \sim-1.7$ dex.

Conclusions. The metallicity of the LMC, as traced by different populations, bears the signature of two major star forming episodes: the first one constituting a thick disc/halo population and the second one a thin disc and bar due to a close encounter with the Milky Way and SMC. The $[\mathrm{Fe} / \mathrm{H}]$ of the recent episode supports an LMC origin for the Stream. The metallicity of the SMC supports star formation, $\sim 3 \mathrm{Gyr}$ ago, as triggered by LMC interaction and sustained by the bar in the outer region of the galaxy. The SMC [Fe/H] agrees with the present-day abundance in the Bridge and shows no significant gradient. The metallicity of M33 supports an "insideout" disc formation via accretion of metal poor gas from the interstellar medium.
\end{abstract}

Key words. galaxies: abundances - Magellanic Clouds - Local Group - stars: AGB and post-AGB - galaxies: stellar content galaxies: individual: M33

\section{Introduction}

The Magellanic Clouds and M33 galaxies are members of the Local Group. They contain many AGB stars that have been used to study their star formation history (SFH) and structure (e.g. Cioni et al. 2000a; van der Marel \& Cioni 2001; Cioni \& Habing 2003; Cioni et al. 2006a,b, 2008a). AGB stars exist in two forms: carbon-rich (C-type) and oxygen-rich (M-type), depending on the chemical abundance (atoms and molecules) of their atmosphere. Their ratio, the $\mathrm{C} / \mathrm{M}$ ratio, is an established indicator of metallicity; the most comprehensive, whilst not perfect, calibration as a function of $[\mathrm{Fe} / \mathrm{H}]$ is given by Battinelli \& Demers (2005).

The investigation of the metallicity gradient in galaxies is directly linked to their formation mechanism. Generally, in a collapse scenario gas is accreted and falls into the centre where stars form, enriching the pre-existing gas. Stars may also form during the accretion process at a given distance from the centre. Bar, disc and halo components as well as the dynamical interaction of galaxies and the accretion of satellites alter the distribution of gas. The detection of metallicity and age gradients is crucial to interpret the formation and evolution mechanisms.

Gradients in total metallicity, iron, oxygen or $\alpha$ elements may differ because they do not share the same origin and evolution, and therefore trace different moments in the history of galaxies. According to stellar evolution theory, iron-peak elements are mostly produced in the explosion of supernovae $(\mathrm{SNe})$ of type I, with low- and intermediate-mass star progenitors. Oxygen and other $\alpha$ elements are instead primarily produced by
SNe type II, with massive star progenitors. The relation between iron and oxygen depends on the galaxy.

The Magellanic Clouds are a pair of interacting galaxies. Recent measurements of their proper motion (Kallivayalil et al. 2006a,b) suggest that they are approaching the Milky Way (MW) for the first time (Besla et al. 2007). Their mutual interaction, rather than the interaction with the MW, is fundamental in shaping their SFH and metallicity gradients. The large magellanic cloud (LMC) is a late-type spiral galaxy seen nearly face-on, rich in gas and with active star formation while the small magellanic cloud (SMC) is a highly inclined irregular galaxy with less active star formation. Their dynamical interaction is claimed to be responsible for the various star forming episodes and of the creation of the Magellanic Bridge, connecting the two galaxies, (Gordon et al. 2009) and the Stream (Nidever et al. 2008). The LMC is probably just a few kpc thick, along the line of sight, but the SMC has a more complex structure that may extend up to $20 \mathrm{kpc}$. Their apparent morphology is dominated by the distribution of young stars, while evolved stars trace a more regular elliptical structure (Cioni et al. 2000a). Embedded in each galaxy is a bar. The Magellanic Clouds have experienced an extended SFH (e.g. Hill 2000; Zaritsky et al. 2002; 2004; Cole et al 2005; Pompéia et al. 2008; Gallart et al. 2008; Carrera et al. 2008a,b).

M33 is an isolated spiral galaxy. Its most prominent feature is a warped disc embedding well delineated spiral arms. Surface brightness profiles indicate that the disc is truncated at $\sim 8 \mathrm{kpc}$ (Ferguson et al. 2007) and while there might be a halo (Schommer et al. 1991; McConnachie et al. 2006a; Sarajedini et al. 2006), there is no bulge (McLean \& Liu1996). The SFH 
of the inner disc is different from that of the outer disc/halo (e.g. Barker et al. 2007; Williams et al. 2009). Inhomogeneities in age and metallicity have been presented by Cioni et al. (2008a).

This paper, motivated by previous investigations, explores the $[\mathrm{Fe} / \mathrm{H}]$ abundance variation with galactocentric distance in the Magellanic Clouds and M33. Section 2 describes the AGB samples, the calculation of $[\mathrm{Fe} / \mathrm{H}]$ and of distances as well as of gradients. Section 3 discusses these gradients with respect to the literature and the implication on the structure, formation and evolution of each galaxy. Section 4 concludes this study. The appendix discusses the iron abundance with respect to the Ca II triplet (Appendix A) and the $\mathrm{C} / \mathrm{M}$ ratio (Appendix B).

\section{Analysis}

\subsection{The AGB sample}

The data analysed here are from Cioni \& Habing (2003), for the Magellanic Clouds, and Cioni et al. (2008a), for M33. The samples contain 32801 and 7653 AGB stars within two areas of $20 \times 20 \mathrm{deg}^{2}$ in the LMC and SMC, respectively, and 14360 in M33 within $1.8 \times 1.8 \mathrm{deg}^{2}$. The LMC and SMC areas, centred at $\left(\alpha=82.25^{\circ}, \delta=-69.5^{\circ}\right)$ and $\left(\alpha=12.5^{\circ}, \delta=-73^{\circ}\right)$, were divided using a grid of $100 \times 100$ cells of size $0.04 \mathrm{deg}^{2}$ each. The M33 area, centred at $\left(\alpha=23.46^{\circ}, \delta=30.66^{\circ}\right)$, was divided using a grid of $36 \times 36$ cells of size $0.0025 \mathrm{deg}^{2}$ each.

The number of C- and M-type AGB stars was selected using colour-magnitude diagrams. DENIS- $I J K_{\mathrm{s}}$ data were used for the Magellanic Clouds. This combination of optical and near-infrared broad-band observations allows us to minimize the contamination of the AGB sample by foreground and red giant branch (RGB) stars. The reader should refer to Cioni \& Habing (2003) for the sub-type of AGB stars selected. Only $J K_{\mathrm{s}}$ data were available for M33 and the most reliable, whilst not complete, sample of AGB stars was obtained by selecting AGB stars above the tip of the RGB that were classified as $\mathrm{C}$ if $\left(J-K_{\mathrm{S}}\right)_{0}>1.36$ and as $\mathrm{M}$ if comprised within two slanted lines, following the shape of the giant branch and in agreement with theoretical stellar evolutionary models (see Cioni et al. 2008a).

Prior to the selection of the AGB sample, the data were dereddend to account for interstellar extinction along the line of sight, as in Cioni et al. (2006a,b, 2008a). If differential reddening is, however, present throughout a galaxy, this may affect the source selection based on colours and magnitudes. The extinction map derived by Zaritsky et al. (2004) for the LMC shows that dust is highly localized near young and hot stars while there is no global pattern. The extinction towards older stars is bimodal, reflecting the location of stars in front of and behind a thin dust layer embedding the young stars. This extinction corresponds to absorption peaks of $A_{J}=0.02,0.12$ and $A_{K}=0.01$, 0.05 for $A_{V}=0.1,0.5$ and adopting the Glass \& Schultheis (2003) extinction law. The lack of pattern does not influence the global shape of the galactocentric trends discussed here, but it will introduce scatter around them. No extinction map is available at present for the SMC and M33.

\subsection{The iron abundance}

The $\mathrm{C} / \mathrm{M}$ ratio has been established as a good indicator of metallicity. Battinelli \& Demers (2005) have provided a relation to convert this ratio into iron abundance: $[\mathrm{Fe} / \mathrm{H}]=-1.32 \pm$ $0.07-0.59 \pm 0.09 \times \log (\mathrm{C} / \mathrm{M} 0+)$. This relation was obtained by homogeneously classifying AGB stars in a wide sample of Local Group galaxies and by adopting $[\mathrm{Fe} / \mathrm{H}]$ values from, mostly,

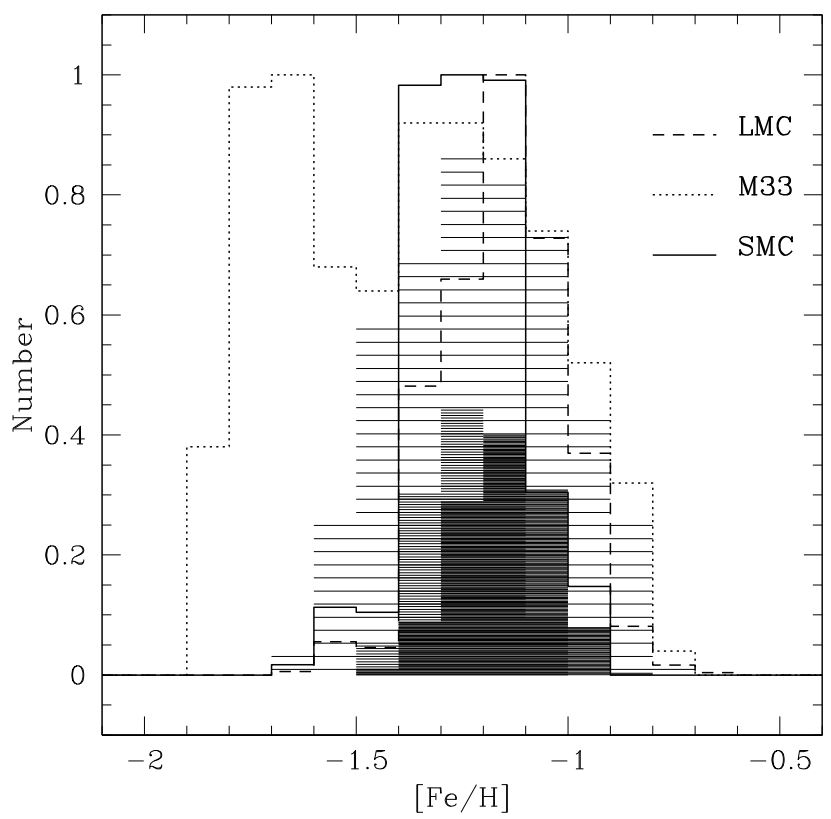

Fig. 1. Metallicity distribution for the LMC (dashed line), M33 (dotted line) and the SMC (continuous line) normalized to their peak of 544,115 and 50 stars, respectively. Shaded areas indicate $[\mathrm{Fe} / \mathrm{H}]$ values with $\sigma_{[\mathrm{Fe} / \mathrm{H}]}<0.2$ dex, different patterns correspond to the LMC (filled lines), SMC (narrow-spaced lines) and M33 (wide-spaced lines).

RGB stars. The latter represent the closest approximation to the metallicity of the AGB progenitors across their age range. A reassessment of this relation, $[\mathrm{Fe} / \mathrm{H}]=-1.39 \pm 0.06-0.47 \pm 0.10 \times$ $\log (\mathrm{C} / \mathrm{M} 0+)$, is given in Appendix B.

The $\mathrm{C} / \mathrm{M}$ ratio obtained within each cell across the Magellanic Clouds and M33 has been converted into $[\mathrm{Fe} / \mathrm{H}]$ using this relation. The uncertainty in the resulting values of $[\mathrm{Fe} / \mathrm{H}]$ is the propagated error on the parameters that characterize the relation and on the error on the number of $\mathrm{C}$ and $\mathrm{M}$ stars, the square root of these numbers. This error is $\geq 0.1$ if $\mathrm{C} / \mathrm{M} \geq 0.2$ or $[\mathrm{Fe} / \mathrm{H}] \leq 1.4$ dex. Figure 1 shows the metallicity distribution across each galaxy. The distribution of M33 is bimodal with peaks at $[\mathrm{Fe} / \mathrm{H}] \approx-1.3$ and $[\mathrm{Fe} / \mathrm{H}] \approx-1.65$ dex. The LMC peaks at $[\mathrm{Fe} / \mathrm{H}] \approx-1.15 \mathrm{dex}$ and the $\mathrm{SMC}$ at $[\mathrm{Fe} / \mathrm{H}] \approx-1.25 \mathrm{dex}$. Sources with $\sigma_{[\mathrm{Fe} / \mathrm{H}]}<0.2$ dex populate mostly the metal-rich peak in M33; the metal-poor peak appears, however, significant with respect to the uncertainties involved. The metallicity of the LMC is higher than that of the SMC while the metal-rich M33 peak is wide and encompasses both Magellanic peaks.

\subsection{The AGB gradient}

A galactocentric distance, $R_{\mathrm{GC}}$, has been associated with each cell. These values were derived using distance $(D)$, position angle of the major axis (PA) and inclination $(i)$, as listed in Table 1 and proceeding as follows:

- convert the equatorial coordinates of each star $\left(\alpha_{i}, \delta_{i}\right)$ into angular coordinates $\left(x_{i}, y_{i}\right)$;

- group stars into cells of a given grid (Sect. 2.1);

- rotate the coordinate system according to:

$$
\begin{aligned}
& x_{1}=x \times \cos (\theta)+y \times \sin (\theta) \\
& y_{1}=y \times \cos (\theta)-x \times \sin (\theta)
\end{aligned}
$$

where $(x, y)$ are mid-cell values in $\operatorname{deg}$ and $\theta=\mathrm{PA}-90^{\circ}$; 
Table 1. Parameters of galaxy structures.

\begin{tabular}{ccccc}
\hline \hline Galaxy & $\begin{array}{c}\text { Distance } \\
(\mathrm{kpc})\end{array}$ & $\begin{array}{c}\text { Pos. angle } \\
(\mathrm{deg})\end{array}$ & $\begin{array}{c}\text { Inclination } \\
(\mathrm{deg})\end{array}$ & Reference(s) \\
\hline LMC & 51 & 189.3 & 34.7 & $1,5,3$ \\
SMC & 63 & 45 & 65.5 & 1,4 \\
M33 & 840 & 22 & 53 & 2 \\
\hline
\end{tabular}

(1) Cioni et al. (2000b); (2) Magrini et al. (2009); (3) van der Marel \& Cioni (2001); (4) Paturel et al. (2003); (5) van der Marel (2001).

- de-project using:

$$
y_{2}=y_{1} / \cos (i)
$$

- calculate the angular distance and convert into kpc with:

$$
\begin{aligned}
& d_{\mathrm{deg}}=\sqrt{x_{1}^{2}+y_{2}^{2}} \\
& d_{\mathrm{kpc}}=D \times \operatorname{tg}(d)
\end{aligned}
$$

where $d$ is the angular distance of each cell.

No uncertainty was calculated for the resulting $R_{\mathrm{GC}}$ values due to the unknown uncertainty on the parameters that describe the structure of the galaxies. It is likely that the uncertainty on $R_{\mathrm{GC}}$ will be dominated by the uncertainty on the distance to the galaxy, especially where the thickness along the line of sight is not known, e.g. for the SMC. The distribution of $[\mathrm{Fe} / \mathrm{H}]$ is shown in Figs. 2-4 for the LMC, SMC and M33, respectively.

Figure 2 shows that the LMC has a smooth gradient where the central region is more metal rich, $[\mathrm{Fe} / \mathrm{H}] \sim-1.0 \mathrm{dex}$, compared to the outer region, $[\mathrm{Fe} / \mathrm{H}] \sim-1.5 \mathrm{dex}$ at $\sim 10 \mathrm{kpc}$. A non-weighted least square fit through all points gives $[\mathrm{Fe} / \mathrm{H}]=$ $-0.035 \pm 0.002 \times R_{\mathrm{GC}}-1.03 \pm 0.01$ with a typical uncertainty on a single measurement of $0.14(\mathrm{rms})$ while if only points with $\sigma_{[\mathrm{Fe} / \mathrm{H}]}<0.2$ dex are considered, the resulting fit is $[\mathrm{Fe} / \mathrm{H}]=$ $-0.047 \pm 0.003 \times R_{\mathrm{GC}}-1.04 \pm 0.01$ with $\mathrm{rms}=0.09$.

A negligible gradient is derived for the SMC (Fig. 3). A non-weighted least square fit through all points gives $[\mathrm{Fe} / \mathrm{H}]=$ $-0.007 \pm 0.003 \times R_{\mathrm{GC}}-1.22 \pm 0.01$ with $\mathrm{rms}=0.13$ while for $\sigma_{[\mathrm{Fe} / \mathrm{H}]}<0.2$ dex the resulting fit is $[\mathrm{Fe} / \mathrm{H}]=0.004 \pm 0.005 \times$ $R_{\mathrm{GC}}-1.25 \pm 0.01$ with $\mathrm{rms}=0.09$. Note that the slope is different between the two fits but in both cases it is consistent with a flat gradient.

The M33 gradient has a dual distribution (Fig. 4) and the change in slope occurs at about the truncation radius, $\sim 8 \mathrm{kpc}$ (Ferguson et al. 2007). A non-weighted fit of all points with $\sigma_{[\mathrm{Fe} / \mathrm{H}]}<0.2 \mathrm{dex}$, that are approximately confined within this radius and have $[\mathrm{Fe} / \mathrm{H}] \geq-1.5$, gives $[\mathrm{Fe} / \mathrm{H}]=-0.078 \pm 0.003 \times$ $R_{\mathrm{GC}}-0.77 \pm 0.02$ with $\mathrm{rms}=0.11$. Points with $[\mathrm{Fe} / \mathrm{H}]<-1.5 \mathrm{dex}$ have $R_{\mathrm{GC}}>8 \mathrm{kpc}$ and their fit is $[\mathrm{Fe} / \mathrm{H}]=-0.007 \pm 0.002 \times R_{\mathrm{GC}}-$ $1.60 \pm 0.03$ with $\mathrm{rms}=0.09$. By neglecting the dual distribution and the uncertainty one obtains $[\mathrm{Fe} / \mathrm{H}]=-0.057 \pm 0.002 \times R_{\mathrm{GC}}-$ $0.93 \pm 0.02$ with $\mathrm{rms}=0.17$.

\section{Discussion}

\subsection{The large magellanic cloud}

Literature studies of the LMC refer to an inner and an outer disc component simply differentiating how far the observed regions are from the centre. The presence of an inner/outer halo component is instead drawn from metallicity measurements. A halo containing predominantly gas from the initial process of galaxy formation would be metal poorer than the disc of the galaxy where stars have formed. A metal rich halo would instead bear the signature of significant accretion of small bodies. The bar, residing in the disc (Zaritsky et al. 1994), is usually referred to as a separate component and can considerably reduce pre-existing abundance gradients over a few dynamical timescales since its formation (Friedli \& Benz 1995).

\subsubsection{AGB and RGB gradients}

Cole et al. (2005) derived $[\mathrm{Fe} / \mathrm{H}]$ using the $\mathrm{Ca}$ II triplet method in a sample of RGB stars in the LMC bar. Carrera et al. (2008a) used the same method for RGB stars at 3-7 kpc and Pompéia et al. (2008) for RGB stars at $\sim 2 \mathrm{kpc}$. The original data-point from Pompéia et al. (2008) is the mean and standard deviation, $[\mathrm{Fe} / \mathrm{H}]=-0.75 \pm 0.23$, of their sample (Appendix A). The two Cole et al. (2005) points correspond to the disc (metal rich) and to the halo (metal poor). Carrera et al. (2008a) quote metallicities only for the disc. On the other hand, their Fig. 5 shows that a halo component exists in at least two of their fields. I derived the intensity and width of this component from their histograms. Table 2 shows the values of $[\mathrm{Fe} / \mathrm{H}]$ obtained from the Cole et al. (2005), Pompéia et al. (2008) and Carrera et al. (2008a) original data and the values resulting from applying a correction for the difference between $\mathrm{Ca}$ II triplet abundances and abundances obtained directly from iron lines (Appendix A). The latter are used in this study.

Figure 2 shows that RGB values, compared to AGB ones, have a dual behaviour: those of the disc have high abundances and a negligible gradient out to $\sim 6 \mathrm{kpc}$, and those of the halo are metal poor and follow the AGB gradient more closely. The intensity of star forming episodes, the dynamical effect of the bar and the re-distribution of stars from their birth place (Roškar et al. 2008) may be responsible for the different gradients and for the scatter around them. It is unlikely that the photometric criteria used to discriminate between $\mathrm{C}$ - and $\mathrm{M}$-type stars favour low metallicities. In fact it is somewhat easier to distinguish $\mathrm{C}$ stars using their near-infrared colours and magnitudes because at their location there are very few sources of contamination (Cioni et al. 2001; Battinelli \& Demers 2009). The M star region, however, relies strongly on the minimization of the contribution by RGB and galactic dwarf stars. The result is a bias in isolating preferentially metal-rich regions, with low $\mathrm{C} / \mathrm{M}$ ratios, as $\mathrm{M}$ stars would be over-estimated compared to $\mathrm{C}$ stars within the same region.

Most AGB stars are long period variables (LPVs) and Hughes et al. (1991) derived that $40 \%$ of them are old ( $\geq 9$ Gyr) and part of a spheroidal population, with $53 \%$ being of intermediate age $(\sim 4 \mathrm{Gyr})$ and residing in a disc; the others are young $(\leq 1 \mathrm{Gyr})$. Most C stars reside in a thick disc (van der Marel et al. 2002) and are 1-4 Gyr old (Marigo et al. 1999). The distinction between a thin and thick disc is not clear. In our Galaxy, thick disc stars have higher $[\alpha / \mathrm{Fe}]$ than thin disc stars (Soubiran \& Girard 2005) but $[\alpha / \mathrm{Fe}]$ might follow a gradient within either discs (Edvardsson et al. 1993). The low $[\alpha / \mathrm{Fe}]$ ratio measured by Pompéia et al. (2008) suggests a higher contribution by SN type Ia relative to type II, supporting the formation of their stars at intermediate ages. Cole et al. (2000) indicate that these RGB stars are likely 1-3 Gyr old, like most of the RGB stars studied by Cole et al. (2005) and Carrera et al. (2008a). If many of the AGB stars analysed here are older than the bulk of RGB stars, they will more closely follow the outer disc/halo. 


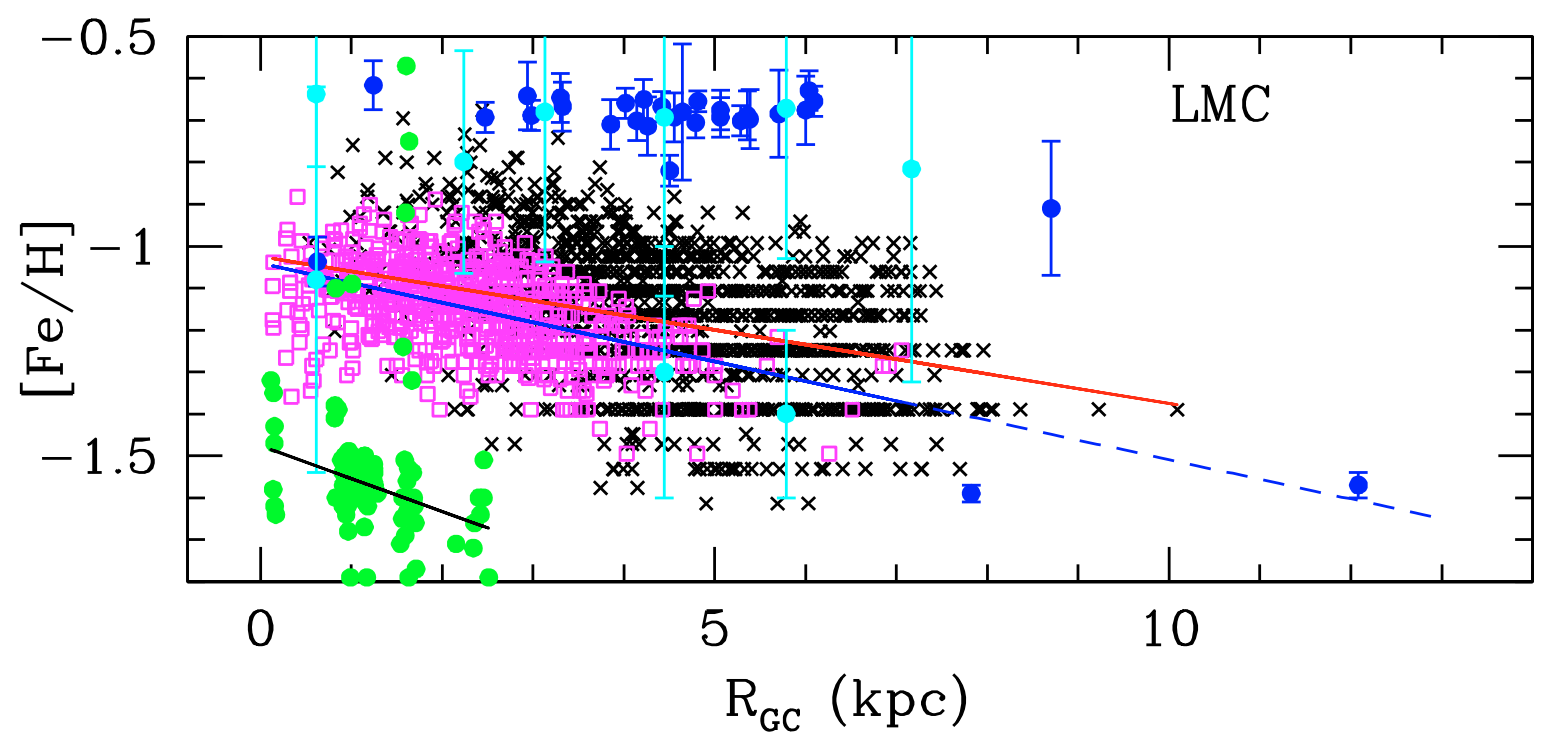

Fig. 2. Iron abundance in the LMC. Points referring to AGB abundances with $\sigma_{[\mathrm{Fe} / \mathrm{H}]}<0.2$ dex are plotted as empty squares (magenta) and those with $0.2 \leq \sigma_{[\mathrm{Fe} / \mathrm{H}]}<0.38$ as crosses (black). The least square fit lines through all data points (red) and through only those with small uncertainties (blue) are indicated, the latter has been prolongued with a dashed line to $13 \mathrm{kpc}$. Filled circles show RGB abundances by Cole et al. (2005), Pompéia et al. (2008) and Carrera et al. (2008a) in the field (light blue) as from Table 2, Grocholski et al. (2006, 2007) for stellar clusters (with small error bars; dark blue) and Borissova et al. (2006) for RR Lyrae stars (without error bars; green), see text for details. The colour figure is available electronically.

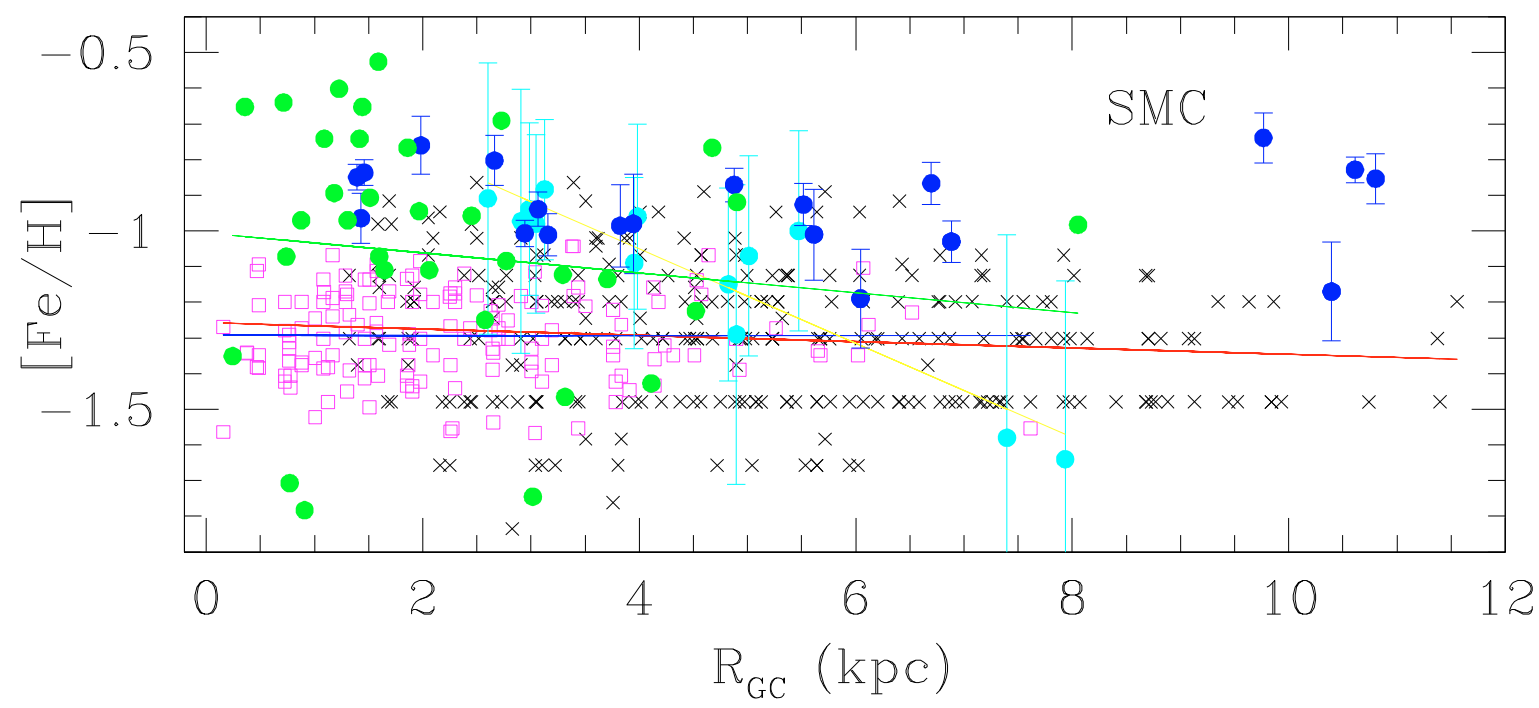

Fig. 3. Iron abundance in the SMC. Points are as in Fig. 2. The least square fit lines through all points (red) and only those with small uncertainties (blue) are indicated. Filled circles with large error bars (light blue) and their least square fit line (yellow) refer to RGB stars by Carrera et al. (2008a) while filled circles with small error bars (dark blue) are for stellar clusters (Parisi et al. 2009; Da Costa \& Hatzidimitriou 1998). Both measurements were corrected as explained in the text. Filled circles without error bars and the best fit line through them (green) are for PNe by Idiart et al. (2007). The colour figure is available electronically.

\subsubsection{Does the LMC have a stellar halo?}

A break in the surface brightness profile, usually associated with the transition between two components, is present at $\sim 4^{\circ}$ ( $\sim 3.6 \mathrm{kpc}$; van der Marel 2001). The AGB gradient at $5<R_{\mathrm{GC}}<$ 8 is only marginally flatter than in the inner disc. Observations of stellar clusters exhibit disc-like kinematics that is very similar to the HI disc (Grocholski et al. 2006, 2007). Their corrected metallicity, using Eq. (A.1), is constant at $[\mathrm{Fe} / \mathrm{H}]=-0.66 \pm 0.02 \mathrm{dex}$, in agreement with the inner disc metallicity in field RGBs. Grocholski et al. (2006) attributed this flattening to the dynamical effect of a bar that occupies a significant fraction of the disc length. The older and metal poorer clusters, however, follow the AGB gradient, regardless of their location with respect to the galaxy centre, this is also true for RGB stars associated with the halo. The small number of these clusters does not allow us to characterize their kinematics but suggests that a halo extends at least to $\sim 14 \mathrm{kpc}$ from the centre; LMC stars have been reported at $\sim 20 \mathrm{kpc}$ (Majewski et al. 2005), while studies of AGB stars are limited to $\sim 10 \mathrm{kpc}$.

RR Lyrae stars are often attributed to the halo of galaxies because of their old age and velocity dispersion (Minniti et al. 2003). In the LMC, they may have formed in the disc and subsequently moved to the halo as a consequence of a merger event 
Table 2. $[\mathrm{Fe} / \mathrm{H}]$ abundances in the LMC

\begin{tabular}{cccc}
\hline \hline $\begin{array}{c}\text { Distance } \\
(\mathrm{kpc})\end{array}$ & $\begin{array}{c}{[\mathrm{Fe} / \mathrm{H}]_{\text {orig }}} \\
(\mathrm{dex})\end{array}$ & $\begin{array}{c}{[\mathrm{Fe} / \mathrm{H}]_{\text {cor }}} \\
(\mathrm{dex})\end{array}$ & Reference \\
\hline 0.6 & $-0.37 \pm 0.15$ & $-0.64 \pm 0.17$ & 1 \\
0.6 & $-1.08 \pm 0.46$ & & 1 \\
2.4 & $-0.75 \pm 0.23$ & $-0.79 \pm 0.27$ & 2 \\
3.1 & $-0.47 \pm 0.31$ & $-0.68 \pm 0.36$ & 3 \\
4.4 & $-0.50 \pm 0.37$ & $-0.69 \pm 0.43$ & 3 \\
4.4 & $-1.30 \pm 0.30$ & & 3 \\
5.8 & $-0.45 \pm 0.31$ & $-0.67 \pm 0.36$ & 3 \\
4.4 & $-1.30 \pm 0.30$ & & 3 \\
7.2 & $-0.79 \pm 0.44$ & $-0.82 \pm 0.50$ & 3 \\
\hline
\end{tabular}

(1) Cole et al. (2005); (2) Pompéia et al. (2008); (3) Carrera et al. (2008a).

in the early formation of the galaxy (Subramaniam 2006). This halo formed before the disc currently traced by red clump giant stars (Subramanian \& Subramaniam 2009). The metallicity of RR Lyrae stars measured by Borissova et al. (2006) and their least square fit, $[\mathrm{Fe} / \mathrm{H}]=-0.078 \pm 0.007 \times R_{\mathrm{GC}}-1.48 \pm 0.03$ with $\mathrm{rms}=0.18$, are shown in Fig. 2. This gradient is steeper than that obtained from AGB stars and population II clusters ( $\geq 9$ Gyr old). The latter is steeper than that from disc RGB stars and intermediate-age (1-3 Gyr) clusters.

\subsubsection{Chemical enrichment and dynamics}

The difference between the AGB and the RR Lyrae stars gradients, supported by old clusters, suggests that chemical enrichment has occured between the formation of their progenitors. If these AGB stars are old (Hughes et al. 1991) a 2 Gyr difference would produce an enrichment of $\sim 0.02 \mathrm{dex} \mathrm{kpc}^{-1} \mathrm{Gyr}^{-1}$. On the other hand, the mean age difference between RR Lyrae stars and young RGB stars or young clusters is $8 \mathrm{Gyr}$. By comparing their gradients we obtain an enrichment of $\sim 0.01 \mathrm{dex} \mathrm{kpc}^{-1} \mathrm{Gyr}^{-1}$. If the enrichment took place in the last $3 \mathrm{Gyr}$ then the rate can be as high as $0.05 \mathrm{dex} \mathrm{kpc}^{-1} \mathrm{Gyr}^{-1}$. A steep enrichment is also supported by the difference between the AGB and young-RGB gradients. The metallicity offset between RR Lyrae stars and AGB stars is comparable to the difference between AGB stars and young RGB stars. This effect can be explained in terms of age differences but could also be influenced by the dispersion in the calibration of the $\mathrm{C} / \mathrm{M}-[\mathrm{Fe} / \mathrm{H}]$ relation as well as uncertainties in metallicities obtained via other methods. There are insufficient data on $\mathrm{HII}$ regions and $\mathrm{PNe}$ to investigate further the chemical enrichment of the LMC. The census of these objects is incomplete and biased towards younger members (Reid \& Parker 2006; Leisy \& Dennefeld 2006; Dufour et al. 1984). Metal poor PNe distributed over a halo fall below the sensitivity of previous studies. High PNe abundances, that do not include iron, are confined to the bar and south eastern region of the LMC, indicating places were star formation was recently active (Leisy \& Dennefeld 2006).

According to Bekki et al. (2004), the LMC experienced a close encounter with the SMC $\sim 4$ Gyr ago. This event caused a new episode of star formation in both the field and cluster population as well as the formation of the LMC bar and Magellanic Stream. The average metallicity of the Stream is $[\mathrm{Fe} / \mathrm{H}]=-0.6 \pm 0.2 \mathrm{dex}$ (Wakker 2001), suggesting a similar age to that of disc RGB and cluster stars (Fig. 2). Nidever et al. (2008) argue for an LMC origin of the Stream that is $\sim 2 \mathrm{Gyr}$ old. This is consistent with ram-pressure gas stripping from the outer LMC disc due to a close passage by the MW (Mastropietro 2008). The new LMC orbit, derived from the new proper motion (Kallivayalil et al. 2006a), implies that the LMC passed perigalacticon $\sim 1.78$ Gyr ago. The AGB gradient analysed here bears the signature of the star forming episode, several Gyr ago, responsible for the formation of a thick disc/halo component. The metal-rich RGB and the cluster stars are the product of this recent episode while RR Lyrae stars, some RGB stars and clusters result from the earliest episode.

\subsection{The small magellanic cloud}

\subsubsection{Flat gradient or metal-rich ring?}

In Cioni et al. (2006b) it was recognized that the SMC bar region is surrounded by a metal-rich ring with signatures of dynamical evolution, moving clumps, as a function of time. This behaviour was un-explained and attributed to the unknown geometry of the galaxy. A ring feature occurring at $\sim 2.5 \mathrm{Gyr}$ and persisting until $\sim 1.6$ Gyr was found by Harris \& Zaritsky (2004) in their SFH analysis. Its age agrees with the age of AGB stars, 0.6-2 Gyr old (Cioni et al. 2003). Before and after, star formation occurred in the bar. In both studies, the metallicity was derived from stellar evolution models in terms of $\mathrm{Z}$ that represents the total heavy element abundance. A flat $[\mathrm{Fe} / \mathrm{H}]$ gradient, then, would be consistent with an $\alpha$ - or O-rich ring.

Very recently, Gonidakis et al. (2009) suggested that the old (K, M and faint $\mathrm{C}$ stars) stellar population rotates and resides on a disc. Combining this information with a central region that started to form stars $\sim 10 \mathrm{Gyr}$ ago it is possible that a metal rich ring is the result of star formation induced by a rotating bar that sustains gas in the outer parts of the galaxy. The rotation speed of the bar may be responsible for an age gradient in the ring. The presence of a bar is also consistent with a flat metallicity gradient because it acts against a linearly decreasing gradient that would instead be present in a bar-less disc galaxy (Martin \& Roy 1994). A bar, regardless of the overall structure of the galaxy (spiral, spheroidal) always resides in a disc (Zaritsky et al. 1994). On the other hand, the investigation by Subramaniam \& Subramanian (2009) shows that the SMC might host a bulge where metal poor and metal rich stars coexist and trace a similar line of sight depth. Although the two concepts do not exclude each other (e.g. Galactic bulge), more evidence is needed to confirm these substructures.

\subsubsection{Halo and chemical enrichment}

The data analysed here do not show evidence for a halo population traced by AGB stars. In the literature there is no evidence for such a halo, although there have been a limited number of studies suggesting that the extent of the SMC disc is greater than the more familiar optical appearance of the galaxy (e.g. Nöel \& Gallart 2007). There is also an apparent lack of metallicity determinations in RR Lyrae stars, usually populating halos.

The $[\mathrm{Fe} / \mathrm{H}]$ distribution of stellar clusters (Parisi et al. 2009; Da Costa \& Hatzidimitriou 1998) also shows a flat gradient. The cluster metallicities plotted in Fig. 3 are those obtained after applying Eq. (A.1) to the original data according to Appendix A. The correction might not be appropriate for the Da Costa \& Hatzidimitriou (1998) values that rely on a different calibration but their agreement with the Parisi et al. (2009) measurements is independent of this correction. Most clusters are of intermediate age, $2.7 \pm 1.6$ Gyr. There are only three old clusters, $\geq 9$ Gyr, similar to those found in the metal poor outer disc/halo of the 


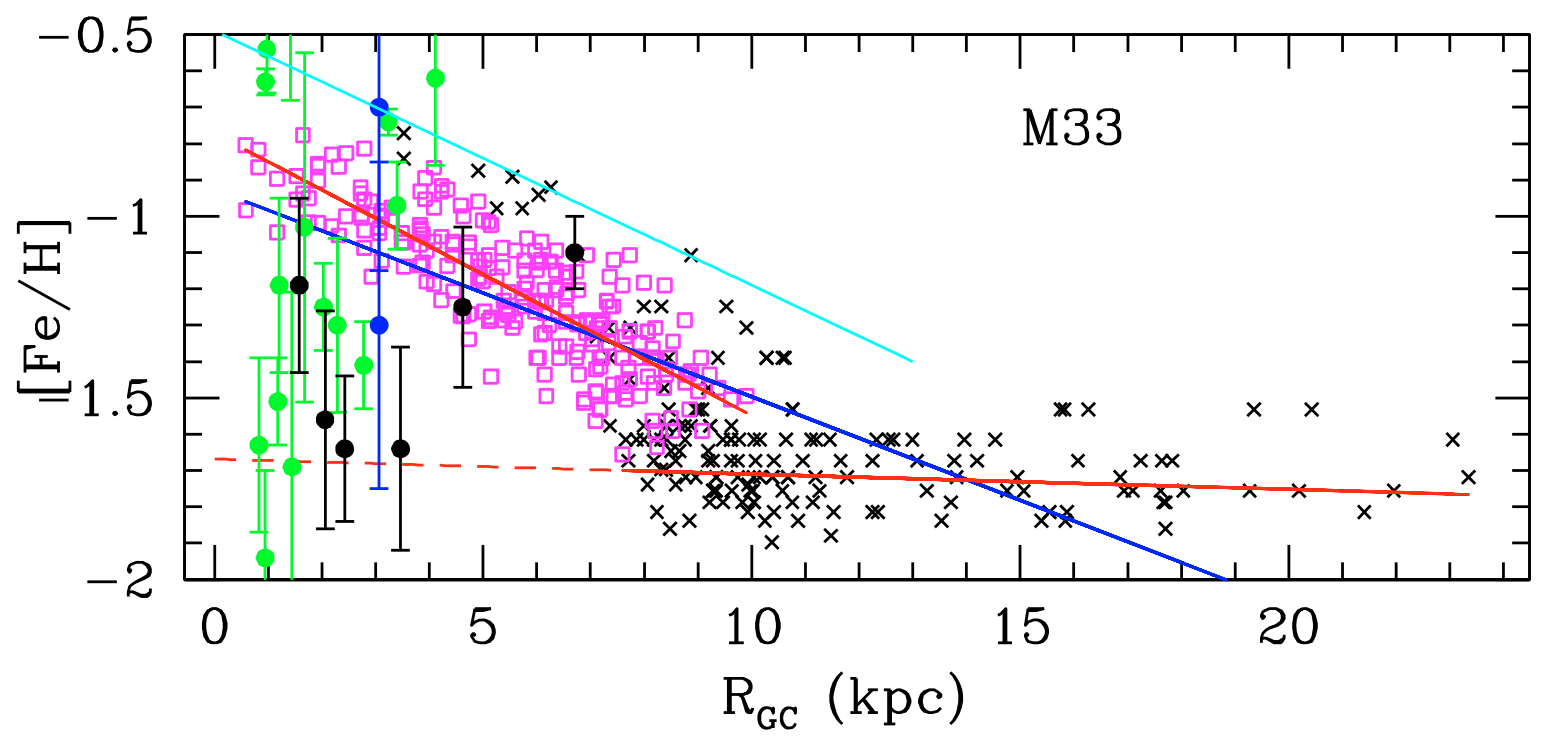

Fig. 4. Iron abundance in M33. Points are as in Fig. 2. The least square fit line thorough all points (blue) and those with small uncertainties (red) are indicated. Magenta points are confined within $\sim 8 \mathrm{kpc}$, the truncation radius of M33 (Ferguson et al. 2007). Beyond this radius and for $[\mathrm{Fe} / \mathrm{H}]<-1.5$ dex the least square fit (red) is shallower; this fit has been prolonged towards the centre of the galaxy with a dashed line. Filled circles (green \& black) show the abundance of stellar clusters attributed to the halo of the galaxy (Ma et al. 2004; Sarajedini et al. 2000) and to the RR Lyrae star population (blue) from Sarajedini et al. (2006). The RGB gradient derived by Barker et al. (2007) is shown in cyan. The colour figure is available electronically.

LMC but their metallicity does not differ from that of younger clusters.

RGB metallicities by Carrera et al. (2008a) suggest a steep gradient, $[\mathrm{Fe} / \mathrm{H}]=-0.13 \pm 0.02 \times R_{\mathrm{GC}}-0.52 \pm 0.08$ with $\mathrm{rms}=$ 0.10 (Fig. 3), after applying Eq. (A.1) to those fields dominated by a stellar population younger than 5 Gyr. The two fields that drive the steepness of the gradient might not be representative of all position angles at similar de-projected radii in the SMC and have very large error bars. A fit that excludes these points is shallower, $[\mathrm{Fe} / \mathrm{H}]=-0.08 \pm 0.03 \times R_{\mathrm{GC}}-0.71 \pm 0.11$ with $\mathrm{rms}=0.08$. The difference betwen the AGB gradient and the gradient from young RGB stars implies a chemical enrichment of $0.04 \mathrm{dex} \mathrm{kpc}^{-1} \mathrm{Gyr}^{-1}$ for an average age difference of $2 \mathrm{Gyr}$.

Idiart et al. (2007) measured $[\mathrm{O} / \mathrm{H}]$ in many PNe and converted it into $[\mathrm{Fe} / \mathrm{H}]$ using: $[\mathrm{Fe} / \mathrm{H}]=0.16+1.27 \times[\mathrm{O} / \mathrm{H}]$. By de-projecting the coordinates of each $\mathrm{PNe}$ as in this study, a gradient of $-0.03 \pm 0.05$ dex is obtained (Fig. 3). These PNe are mostly located within $5 \mathrm{kpc}$ except one at $8 \mathrm{kpc}$. It is interesting to note that with the solar abundance calibration by Asplund et al. (2004) PNe are metal-richer than AGB stars by $~ 0.3$ dex while with the Anders \& Grevesse (1989) calibration there is good agreement. In the calculation of the PNe gradient I excluded objects with depressed oxygen abundance and four others for which I could not find coordinates. Figure 3 shows $39 \mathrm{PNe}$, with no distinction between type I and II, and their metallicities are consistent with a flat gradient, but it is somewhat steeper than that obtained from AGB stars. AGB stars are the precursors of PNe and the age difference between the two is on average negligible. Known PNe are, however, biased towards the largest, most asymmetric and luminous members, that have intermediate-mass stars progenitors (Jacoby \& De Marco 2002). In addition, the initial metallicity derived from the oxygen abundance of $\mathrm{PNe}$ is questionable (Leisy \& Dennefeld 2006) and it would be more appropriate to use elements that are not modified during the AGB evolution. In the SMC only a small number of HII regions have been observed with the aim of determining their chemical abundance (Dufour 1984). Their $[\mathrm{O} / \mathrm{H}]$ abundance agrees with that measured in PNe and shows a small scatter, 0.08 dex. A larger sample of both HII regions and $\mathrm{PNe}$ is needed before relating them to the chemical enrichment of the galaxy. Reid \& Parker (2006) have shown, for the LMC, that many PNe await to be discovered.

\subsubsection{Dynamics and the magellanic bridge}

The age-metallicity relation as measured in different SMC fields (Carrera et al. 2008a) suggests that after an initial episode of gas enrichment ( $\leq 7$ Gyr ago; Tosi et al. 2008), the SMC experienced a period of quiescent evolution before a new episode $\sim 3$ Gyr ago. At that time, the SMC had a close encounter with the LMC (Piatti et al. 2005, Bekki et al. 2004). Was this episode responsible for the formation of the Magellanic Bridge? The present-day metallicity of the Bridge, from early-type stars, is $[\mathrm{Z} / \mathrm{H}]=-1.05 \pm 0.06$ dex (Lehner et al. 2008), where $\mathrm{Z}$ includes oxygen, nitrogen and argon abundances. This value is in good agreement with the mean metallicity shown in Fig. 3, supporting an SMC origin for the Bridge dating back a few Gyrs. The $[\mathrm{Fe} / \mathrm{H}]$ found for the LMC AGBs is similar to the SMC ones and it is not excluded that the Bridge also has been influenced by LMC material at an earlier epoch.

Star formation in the Bridge started 100-200 Myr ago (Harris 2007), when the LMC and the SMC had another close encounter (Kallivayalil et al. 2006a). This is the same time at which the SMC tail, a tail of tidal origin located within the Bridge, was stripped from the galaxy (Gordon et al. 2009). This means that if the Bridge material were stripped earlier, it did not form stars. This scenario would support the very low metal abundances derived by Dufton et al. (2008), $[\mathrm{Fe} / \mathrm{H}]-1.73 \pm 0.15 \mathrm{dex}$ from B-type stars. This value is much lower than any of the values in Fig. 3. Note that the LMC metallicity at $\sim 5 \mathrm{kpc}$ from the centre, close to the break radius, corresponds to the metallicity of the SMC. Was the LMC gas accreted from the SMC via the Bridge? In the interaction process between the galaxies it is not unreasonable to expect that the smaller galaxy will suffer 
more severe disruption in its outer parts than the largest galaxy. On the other hand, the outer parts of the largest galaxy will be more loosely bound than its central parts and prone to disruption. A more systematic investigation of the stellar population in the Bridge and in the outer region of the Magellanic Clouds will provide a clearer view of the history of their interaction.

\section{3. $M 33$}

The metallicity distribution of M33 is bimodal and the dual gradient suggests that the galaxy has two clearly distinct components, an inner disc with a typical linearly decreasing gradient away from the centre, up to $\sim 9 \mathrm{kpc}$, and an outer disc/halo population that dominates beyond this distance with a much shallower gradient. Barker et al. (2007) tentatively conclude that beyond $50^{\prime}(\sim 13 \mathrm{kpc})$ the metallicity gradient flattens but the analysis supporting this behaviour (Brooks et al. 2004, Davidge 2003) was highly contaminated by foreground galactic stars and background galaxies. Ferguson et al. (2007) identified a break in the surface brightness profile of M33 at $\sim 8 \mathrm{kpc}$ that nicely corresponds with the change in the slope of the AGB gradient, suggesting the existence of two components. The analysis of stellar clusters (Sarajedini et al. 2000, Ma et al. 2004) and RR Lyrae stars (Sarajedini et al. 2006) support this scenario. Some clusters de-project onto the inner disc gradient while others do agree, within the errors, with an outer disc/halo metallicity. Furthermore, Chandar et al. (2002), by analysing the kinematics of clusters, concluded that old halo candidates have an $[\mathrm{Fe} / \mathrm{H}]$ range between -1.0 and -2.0 . This is consistent both with the plateau derived here beyond $\sim 8 \mathrm{kpc}$ and with the metalpoor peak of the metallicity distribution of the Galaxy's globular clusters (Armandroff 1989).

\subsubsection{Chemical enrichment and dynamics}

The RGB gradient corresponds to $-0.07 \mathrm{dex} \mathrm{kpc}^{-1}$ (Kim et al. 2002; Tiede et al. 2004; Barker et al. 2007), comparable with that derived from AGB stars. If no distinction is made between inner disc and outer disc/halo AGB stars (Fig. 4), a gradient of $-0.057 \pm 0.002$ is obtained; this is $\sim 0.01$ dex shallower than the RGB gradient. Considering that AGB stars span a large range of ages, this indicates that the galaxy did not experience significant metal enrichment between several to a few Gyr ago. If AGB stars were younger than the RGB stars, they would provide evidence for a flattening of the metallicity gradient with time or viceversa. The latter might explain the offset between the RGB and AGB gradients, but it may also be influenced by the dispersion in the $\mathrm{C} / \mathrm{M}-[\mathrm{Fe} / \mathrm{H}]$ relation.

Recent measurements of the gradient from HII regions (Magrini et al. 2007; Rosolowsky \& Simon 2008) point to a flat gradient, $[\mathrm{O} / \mathrm{H}] \sim-0.03 \mathrm{dex} \mathrm{kpc}^{-1}$, that produces a similar trend in $[\mathrm{Fe} / \mathrm{H}]$ using King (2000) conversion. HII regions trace the present-day star formation and the difference between their gradient and that of the certainly older AGB stars indicates a flattening of the metallicity gradient with time. Magrini et al. (2009) found that HII regions and PNe follow the same [O/H] gradient. On the one hand, galaxy chemical evolution models indicate a steeper gradient for iron than for oxygen simply because iron comes predominantly from slowly evolving SN type I that compared to SN type II have not yet enriched the outer parts of galaxies. On the other hand, the similarity between the $\mathrm{HII}$ regions and $\mathrm{PNe}$ gradients may suggest that the $\mathrm{PNe}$ sample
( 70 older than $0.3 \mathrm{Gyr}$ ) is on average younger than the AGB population $(\sim 14000)$ in the disc.

Williams et al. (2009) have shown that the age of the population in the inner disc decreases radially contrary to the trend in the outer disc (Barker et al. 2007; Cioni et al. 2008a). This provides evidence for an inside-out formation scenario for the M33 disc also supported by simulations. How does the metallicity gradient fit into this picture? Since the majority of the stars near the centre of the disc had formed by $z=1$ and the bulk of the stars farther out formed later (Williams et al. 2009), more time was available to enrich the gas in the centre than in the outer part; heavy elements are also found in the centre because the potential is stronger. The peak of star formation responsible for younger ages at about the truncation radius corresponds to 2 Gyr (Williams et al. 2009; Fig. 3). This suggests that AGB stars formed at that time and at that location as a consequence of accretion of metal poor gas, out of gas that was not enriched in iron by previous star forming episodes, in agreement with a linearly decreasing gradient throughout the inner disc region. These AGB stars are, therefore, older than 2 Gyr.

The galactic chemical evolution models for the formation of the M33 disc by Magrini et al. (2007) indicate a steeper metallicity gradient in the centre, $\sim-0.11 \mathrm{dex} \mathrm{kpc}^{-1}$, than in the outer parts of the galaxy, according to a gas accretion model, with an almost constant gas in-fall rate. The models by Chiappini et al. (2001), for the MW, assume two main accretion episodes: the first forming the halo/thick disc and the second forming the thin disc. In their models the disc forms "inside-out", in agreement with the Williams et al. (2009) results. A similar picture was deduced for the formation of the isolated spiral galaxy NGC 300, a member of the Sculptor group and very similar to M33, showing a negative $[\mathrm{Fe} / \mathrm{H}]$ gradient in the disc and a flat or slighly positive gradient in the outer parts (Vlajić et al. 2009). The alternative explanation populates the outer parts with stellar migration, accounting for the strength of spiral waves (Roškar et al. 2008; Sellwood \& Binney 2002).

\section{Conclusions}

This paper derives the metallicity, $[\mathrm{Fe} / \mathrm{H}]$, for a large sample AGB stars in the Magellanic Clouds and M33 and investigates the spatial gradient with respect to the structure and history of each galaxy as well as other indicators. The values for iron abundance depend strongly on the calibration of the $\log (\mathrm{C} / \mathrm{M})-[\mathrm{Fe} / \mathrm{H}]$ relation. The metallicity in this relation is that of the dominant population of RGB stars that represents the closest approximation, or a lower limit, to the metallicity of the AGB progenitors. The relation provided by Battinelli \& Demers (2005) has been revised in this study to: $[\mathrm{Fe} / \mathrm{H}]=-1.39 \pm 0.06-0.47 \pm$ $0.10 \times \log (\mathrm{C} / \mathrm{M} 0+)$. The resulting gradients provide new constraints for theoretical models for the formation and evolution of these galaxies, and for similar systems where stars cannot yet be resolved.

The metallicity of the LMC decreases away from the centre, $[\mathrm{Fe} / \mathrm{H}]=-0.047 \pm 0.003 \times R_{\mathrm{GC}}-1.04 \pm 0.01$. This AGB gradient is somewhat flatter than that derived from RR Lyrae stars, $-0.078 \pm$ $0.007 \mathrm{dex} \mathrm{kpc}^{-1}$, and it is followed by metal-poor stellar clusters and metal-poor RGB stars, supporting an old and extended (up to $14 \mathrm{kpc}$ ) thick disc or halo population. Most RGB stars and stellar clusters are, however, younger and have a constant metallicity, $[\mathrm{Fe} / \mathrm{H}]=-0.66 \pm 0.02$ dex. They probably formed when the LMC interacted with the MW and SMC a few Gyr ago along with the formation of the LMC bar and of the Stream (Nidever et al. 2008). A flattening of the gradient with time is consistent 
with "inside-out" disc formation (Vlajić et al. 2009), while a dual formation scenario for the halo and the disc reproduces the AGB gradient (Chiappini et al. 2001).

The metallicity of the SMC is consistent with a flat distribution. This result is sustained by different stellar indicators: RGB stars, PNe and clusters regardless of their age. Together with an [M/H]-rich ring (Cioni et al. 2006b; Harris \& Zaritsky 2004) and a rotating old stellar population residing on a disc (Gonidakis et al. 2009), they support the idea that gas shocked, during an encounter with the LMC 3 Gyr ago, started to form stars in the outer parts of the galaxy, altering the classical $[\mathrm{Fe} / \mathrm{H}]$ gradient of a bar-less disc galaxy. Furthermore an increase in the $[\alpha / \mathrm{Fe}]$ ratio in the outermost regions of the disc or a flat $[\mathrm{Fe} / \mathrm{H}]$ gradient due to an equal timescale for disc formation versus distance are also possible scenarios (Chiappini et al. 2001). The [Fe/H] abundance of the SMC agrees with the present-day abundance in the Bridge.

The M33 inner disc extends to $\sim 9 \mathrm{kpc}$, in agreement with previous findings, while the outer disc/halo population reaches $\sim 25 \mathrm{kpc}$. The inner disc is characterised by a steep metallicity gradient, $[\mathrm{Fe} / \mathrm{H}]=-0.078 \pm 0.003 \times R_{\mathrm{GC}}-0.77 \pm 0.02$, while in the outer regions it flattens to $\sim-1.7 \mathrm{dex}$. The presence of two distinct components agrees with an "inside-out" galaxy formation scenario such as for the closely related NGC 300 galaxy (Vlajić et al. 2009) and is confirmed by Williams et al. (2009). The AGB gradient is steeper than that from HII regions, supporting this scenario.

The Magellanic Clouds show a different but linked metallicity history influenced by their structure and dynamical interaction. It is easier to interpret the metallicity of M33 that has, instead, evolved in isolation. The observation of the outer disc/halo population of the Magellanic Clouds as traced here by AGB stars is not complete. Large data bases can be exploited to search for AGBs 8-20 kpc from the centre. There are also fewer studies of chemical abundances and kinematics in the SMC than in the LMC (van der Marel et al. 2008).

The upcoming VISTA survey of the Magellanic System (Cioni et al. 2008b) will provide new data to investigate the metallicity evolution as well as the SFH, extinction and structure across the system. The simultaneous VISTA hemisphere survey will cover the outermost regions of the system. These near-infrared surveys will provide targets for measuring abundances with current and future wide-field spectrographs.

Acknowledgements. I warmly thank Marina Rejkuba for a critical reading of the paper, and Sean Ryan, Ralf Napiwotzki and Janet Drew for interesting scientific discussions contributing to its development.

\section{Appendix A: Ca II triplet metallicity correction}

The study by Pompéia et al. (2008) analyses the spectra of 59 field RGB stars deriving chemical abundances of iron, from both FeI and FeII lines, and other $\alpha$ elements. The authors indicate no systematic error for $[\mathrm{FeII} / \mathrm{H}]$ abundances but a systematic error of $\sim 0.1 \mathrm{dex}$ for $[\mathrm{FeI} / \mathrm{H}]$ abundances. Figure A.1 shows the comparison between $[\mathrm{Fe} / \mathrm{H}]$ values from iron lines and from the $\mathrm{Ca}$ II triplet method; the data points are from their Table 2. The least square fit through these points corresponds to:

$\Delta[\mathrm{Fe} / \mathrm{H}]=-0.575 \pm 0.004 \times[\mathrm{Fe} / \mathrm{H}]_{\mathrm{CaT}}+0.48 \pm 0.01$

where $\Delta[\mathrm{Fe} / \mathrm{H}]=[\mathrm{Fe} / \mathrm{H}]_{\mathrm{CaT}}-[\mathrm{Fe} / \mathrm{H}]_{\text {spec }}$ and $\sigma_{[\mathrm{Fe} / \mathrm{H}]}=0.15$ for each point. This equation, that is well defined for $[\mathrm{Fe} / \mathrm{H}]<$ -1.3 dex, has been used to correct $[\mathrm{Fe} / \mathrm{H}]$ values from the $\mathrm{Ca}$ II triplet, based on the same calibration (Carretta \& Gratton 1997).
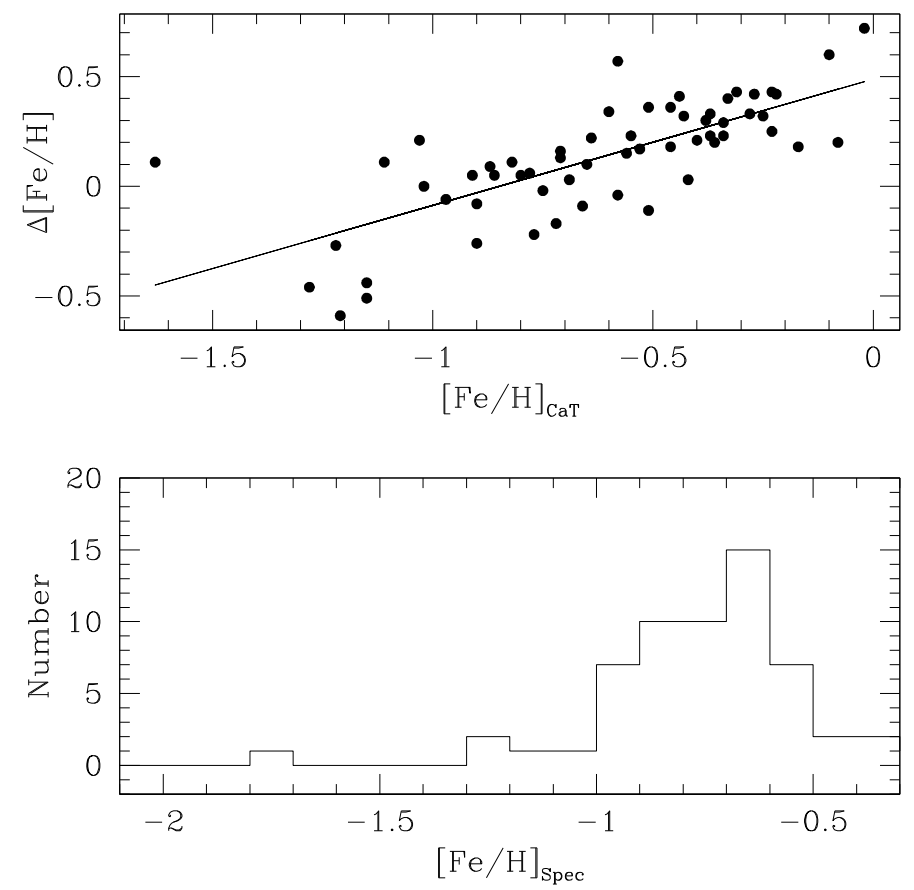

Fig. A.1. (Top) Iron abundance differences derived from the Ca II triplet and direct observation of iron lines as a function of $\mathrm{Ca}$ II triplet values (Pompéia et al. 2008). The line is the least square fit through the data points. (Bottom) Number distribution of iron abundances.

The histogram of iron abundances peaks at a disc stellar population with $[\mathrm{Fe} / \mathrm{H}]_{\mathrm{spec}}=-0.75 \pm 0.23 \mathrm{dex}$.

Compared to Battaglia et al. (2008), who studied RGB metallicities in the Sculptor and Fornax dwarf spheroidal galaxies, Fig. A.1 shows a strong gradient. These authors concluded that Ca II triplet metallicities are overestimated by $\sim 0.1 \mathrm{dex}$ at $[\mathrm{Fe} / \mathrm{H}]<-2.2$ dex, underestimated by $\sim 0.1-0.2$ dex at $[\mathrm{Fe} / \mathrm{H}]>-1.2 \mathrm{dex}$ and have no trend for $[\mathrm{Fe} / \mathrm{H}]>-0.8 \mathrm{dex}$ in the range $-2.5<[\mathrm{Fe} / \mathrm{H}]<-0.5$. Their RGB stars are $>8 \mathrm{Gyr}$ old (Sculptor) and 3-6 Gyr old (Fornax) while LMC RGB stars are mostly young (1-3 Gyr old). Here, Eq. (A.1) is applied only to measurements for RGB stars younger than $\sim 5$ Gyr. A different $\mathrm{SFH}$ also implies a different $\mathrm{Ca} / \mathrm{Fe}$ abundance, therefore, the $\mathrm{Ca}$ II triplet is a good proxy for $[\mathrm{Fe} / \mathrm{H}]$ when the age of RGB stars with respect to the calibrating relation is appropriately considered (Pont et al. 2004).

\section{Appendix B: $\mathrm{C} / \mathrm{M}$ versus $[\mathrm{Fe} / \mathrm{H}]$ calibration}

Battinelli \& Demers (2005) provide a calibration of the metallicity of galaxies versus the $\mathrm{C} / \mathrm{M}$ ratio. The major uncertainty in their relation lies in the values of metallicity adopted from the literature. These values refer to RGB stars, except for IC 10 where $[\mathrm{Fe} / \mathrm{H}]$ was converted from $[\mathrm{O} / \mathrm{H}]$ in $\mathrm{HII}$ regions.

For most of the remaining galaxies, the metallicity was estimated from the colour of the RGB in $(V-I)_{0}$ using the relation by Da Costa \& Armandroff (1990), or Lee (1993), defined at a specific magnitudes below the TRGB. This method is sensitve to metallicity but not to age, that does in fact represent the extent of the RGB population. This section re-assesses the $[\mathrm{Fe} / \mathrm{H}]$ versus $\mathrm{C} / \mathrm{M}$ calibration using updated metallicity measurements for a more homogeneous population. Table B.1 lists the C/M0+ values from Battinelli \& Demers (2005) and the metallicities from different authors. The uncertainty in the metallicity values is the dispersion, $\sigma$, corresponding to half the width of the RGB at the 
Table B.1. RGB metallicity and C/M ratio.

\begin{tabular}{lcccc}
\hline \hline Galaxy & $\begin{array}{c}{[\mathrm{Fe} / \mathrm{H}]} \\
(\mathrm{dex})\end{array}$ & $\begin{array}{c}\sigma \\
(\mathrm{dex})\end{array}$ & C/M0+ & Reference \\
\hline DD0 190 & -2.00 & 0.30 & $2.5 \pm 1.0$ & 1 \\
DDO 210 & -1.30 & 0.27 & $0.09 \pm 0.08$ & 2 \\
IC 1613 & -1.20 & 0.20 & $1.48 \pm 0.31$ & 3 \\
Leo I & -1.52 & 0.20 & $6.5 \pm 6.4$ & 4 \\
M31 & -0.83 & 0.17 & $0.080 \pm 0.004$ & 5 \\
NGC 147 & -1.13 & 0.15 & $0.30 \pm 0.03$ & 6 \\
NGC 185 & -1.11 & 0.15 & $0.24 \pm 0.03$ & 6 \\
NGC 205 & -1.06 & 0.15 & $0.20 \pm 0.01$ & 6 \\
NGC 3109 & -1.70 & 0.30 & $1.85 \pm 0.21$ & 7 \\
NGC 6822 & -1.50 & 0.30 & $0.90 \pm 0.08$ & 8 \\
Pegasus & -1.10 & 0.25 & $0.62 \pm 0.22$ & 9 \\
SagDIG & -2.08 & 0.20 & $4.0 \pm 3.0$ & 10 \\
WLM & -1.45 & 0.30 & $15.0 \pm 7.1$ & 11 \\
\hline
\end{tabular}

(1) Aparicio \& Tikhonov (2000); (2) McConnachie et al. (2006b); (3) Skillman et al. (2003); (4) Tolstoy et al. (2003); (5) Brown et al. (2008); (6) Butler \& Martinez-Delgado (2005); (7) Battinelli \& Demers (2005); (8) Gallart et al. (1996); (9) Aparicio (1994); (10) Momany et al. (2002); (11) Minniti \& Zijlstra (1997).

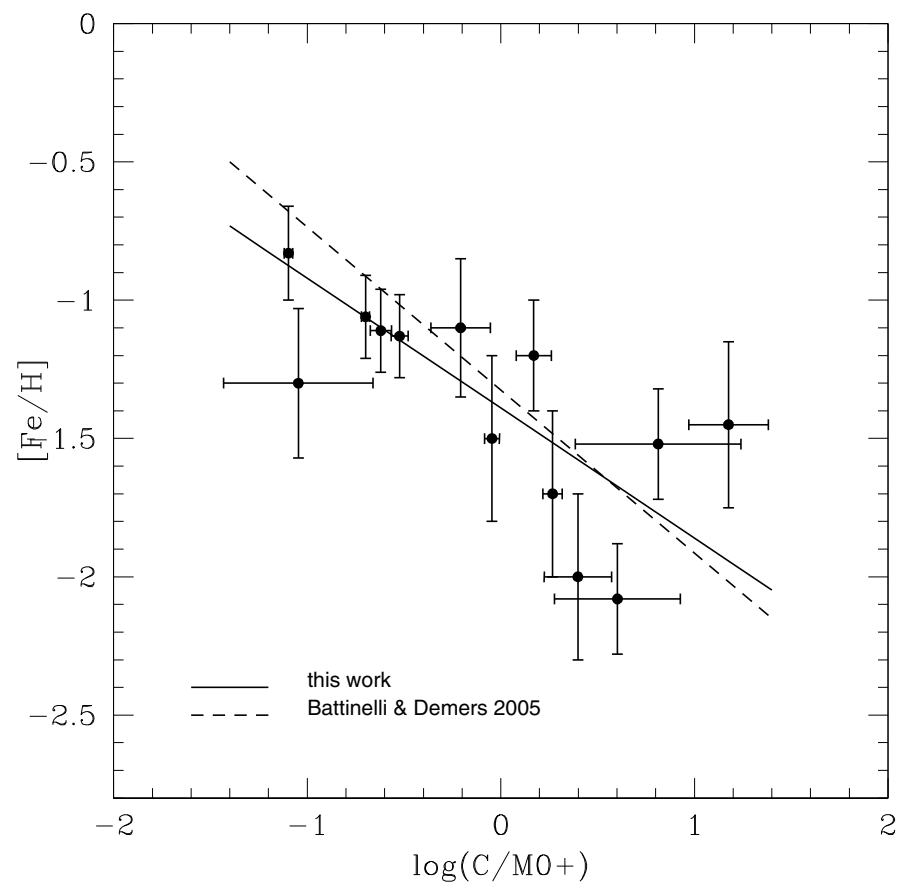

Fig. B.1. Metallicity as a function of $\mathrm{C} / \mathrm{M}$ ratio. Points and their weighted least square fit line (solid line) are for RGB stars as discussed in the text. The dashed line is from Battinelli \& Demers (2005).

calibrating colour. This quantity is a better indicator than the statistical uncertainty of the range of ages for the RGB population within each galaxy.

The RGB parameters for the DDO 190 galaxy, well outside of the Local Group, were obtained by Battinelli \& Demers (2006) for the C/M ratio, and Aparicio \& Tikhonov (2000) for $[\mathrm{Fe} / \mathrm{H}]$. The $[\mathrm{Fe} / \mathrm{H}]$ for NGC 3109 is the mean of the three values quoted in Battinelli \& Demers (2005).

In the case of NGC 6822 , the RGB colour gives a metallicity 0.25 dex lower. Battinelli \& Demers (2005) averaged this value with the value obtained from the RGB slope in the nearIR domain. For DDO 210, McConnachie et al. (2006b) obtained $[\mathrm{Fe} / \mathrm{H}]=-1.3$ dex using the RGB colour method and corresponding to an RGB population $4 \pm 2$ Gyr old. This value is 0.6 dex higher than the value used by Battinelli \& Demers (2005). A difference of $\sim 0.2$ dex is found for NGC147, NGC 185 and NGC 205 by Butler \& Martínez-Delgado (2005) who obtained a mean RGB metallicity of -1.11 dex and -1.06 , respectively.

For IC 1613 the metallicity was derived from the SFH resulting from fitting colour-magnitude diagrams with synthetic diagrams produced using stellar evolution models. These models provide metallicity in terms of the total metallicity, $Z$, that when converted to iron represents an upper limit. The error in $[\mathrm{Fe} / \mathrm{H}]$ given in Table B. 1 coincides with the FWHM of the RGB colour from Bernard et al. (2007). A similar study has been done recently in $\mathrm{M} 31$ resulting in $[\mathrm{Fe} / \mathrm{H}]=-0.83$ dex (Brown et al. 2008). This is a mean value among fields $10-35 \mathrm{kpc}$ from the galaxy centre corresponding to a RGB population with a mean age of $10.4 \pm 0.6$ Gyr.

Among the metallicities used by Battinelli \& Demers (2005), that of Leo I refers to spectroscopic iron lines observed in RGB stars. In the meantime there have been no other direct measures of iron lines in the current sample of Local Group galaxies, but several authors have derived iron from the observation of the Ca II triplet in RGB stars. These measurements are not included here because of two uncertainties: the assumption on the $[\mathrm{Ca} / \mathrm{Fe}]$ abundance ratio, necessary to convert $\mathrm{Ca}$ II triplet abundances to iron, and the age of the RGB stars (Appendix A).

Summarizing, updating metallicities obtained from the colour of the RGB, including those of RGB populations derived from the SFH method (IC 1613 only) and iron lines (Leo I only), and excluding IC 10 gives the following relation:

$[\mathrm{Fe} / \mathrm{H}]=-1.39 \pm 0.06-0.47 \pm 0.10 \times \log (\mathrm{C} / \mathrm{M} 0+)$

with $\chi^{2}=1.1$. Figure B.1 shows the distribution of the RGB points listed in Table B.1, and their weighted least square fit line as well as the line derived by Battinelli \& Demers (2005). Weights account for both the error on the $\mathrm{C} / \mathrm{M} 0+$ and $[\mathrm{Fe} / \mathrm{H}]$.

\section{References}

Anders, E., \& Grevesse, N. 1989, Geochim. Cosmochim. Acta, 53, 197 Aparicio, A. 1994, ApJ, 347, L27

Aparicio, A., \& Tikhonov, N. 2000, AJ, 119, 2183

Armandroff, T. E. 1989, AJ, 97, 375

Asplund, M., Grevesse, N., Suval, A. J., et al. 2004, A\&A, 417, 751 Barker, M. K., Sarajedini, A., Geisler, D., et al. 2007, AJ, 133, 1125 Battaglia, G., Irwin, M., Tolstoy, E., et al. 2008, MNRAS, 383, 183 Battinelli, P., \& Demers, S. 2005, A\&A, 434, 657

Battinelli, P., \& Demers, S. 2006, A\&A, 447, 473

Battinelli, P., \& Demers, S. 2009, A\&A, 493, 1075

Bekki, K., Couch, W. J., Beasley, M. A., et al. 2004, ApJ, 610, L93

Bernard, E. J., Aparicio, A., Gallart, C., et al. 2007, AJ, 134, 1124

Besla, G., Kallivayalil, N., Hernquist, L., et al. 2007, ApJ, 668, 949 Borissova, J., Minniti, D., Rejkuba, M., et al. 2006, A\&A, 460, 459 Brooks, R. S., Wilson, C. D., \& Harris, W. E. 2004, AJ, 128, 237 Brown, T. M., Beaton, M., Chiba, M., et al. 2008, ApJ, 685, L121 Butler, D. J., \& Martínez-Delgado, D. 2005, AJ, 129, 2217

Carretta, E., \& Gratton, R. G. 1997, A\&AS, 121, 95

Carrera, R., Gallart, C., Hardy, E., et al. 2008a, AJ, 135, 836

Carrera, R., Gallart, C., Aparicio, A., et al. 2008b, AJ, 136, 1039

Chandar, R., Bianchi, L., Ford, H. C., et al. 2002, AJ, 564, 712

Chiappini, C., Matteucci, F., \& Romano, D. 2001, ApJ, 554, 1044

Cioni, M.-R. L., \& Habing, H. J. 2003, A\&A, 402, 133

Cioni, M.-R. L., Habing, H. J., \& Israel, F. P. 2000a, A\&A, 358, L9

Cioni, M.-R. L., van der Marel, R. P., Loup, C., et al. 2000b, A\&A, 359, 601

Cioni, M.-R. L., Marquette, J.-B., Loup, C., et al. 2001, A\&A, 377, 945

Cioni, M.-R. L., Blommaert, J. A. D. L., Groenewegen, M. A. T., et al. 2003, A\&A, 406, 51

Cioni, M.-R. L., Girardi, L., Marigo, P., et al. 2006a, A\&A, 448, 77 
Cioni, M.-R. L., Girardi, L., Marigo, P., et al. 2006b, A\&A, 452, 195 Cioni, M.-R. L., Irwin, M., Ferguson, A. M. N., et al. 2008a, A\&A, 487, 131 Cioni, M.-R. L., Bekki, K., Clementini, G., et al. 2008b, PASA, 25, 121 Cole, A. A., Smecker-Hane, T. A., \& Gallagher, J. S. 2000, AJ, 120, 1808

Cole, A. A., Tolstoy, E., Gallagher, J. S., et al. 2005, AJ, 129, 1465

Da Costa, G. S., \& Armandroff, T. E. 1990, AJ, 100, 162

Da Costa, G. S., \& Hatzidimitriou, D. 1998, AJ, 115, 1934

Davidge, T. J. 2003, AJ, 125, 3046

Dufour, R. J. 1984, Structure and evolution of the MC's, ed. S. van den Bergh, \& K.S. de Boer, IAU Symp., 108, 353

Dufton, P. L., Ryans, R. S. I., Thompson, H. M. A., et al. 2008, MNRAS, 385, 2261

Edvardsson, B., Andersen, J., Gustafsson, B., et al. 1993, A\&A, 275, 101

Ferguson, A., Irwin, M., Chapman, S., et al. 2007, Island Universes (ASSP Springer), 239

Friedli, D., \& Benz, W. 1995, A\&A, 301, 649

Gallart, C., Aparicio, A., \& Vilchez, J. M. 1996, AJ, 112, 1928

Gallart, C., Stetson, P. B., Meschin, I., et al. 2008, ApJ, 682, L89

Glass, I. S., \& Schultheis, M. 2003, MNRAS, 345, 39

Gonidakis, I., Livanou, E., Kontizas, E., et al. 2009, A\&A, 496, 375

Gordon, K. D., Bot, C., Muller, E., et al. 2009, ApJ, 690, L76

Grocholski, A. J., Cole, A. A., Sarajedini, A., et al. 2006, AJ, 132, 1630

Grocholski, A. J., Sarajedini, A., Olsen, K., et al. 2007, AJ, 134, 680

Harris, J. 2007, ApJ, 658, 345

Harris, J., \& Zaritsky, D. 2004, AJ, 127, 1531

Hill, V., François, P., Spite, M., et al. 2000, A\&A, 364, L19

Hughes, S. M. G., Wood, P. R., \& Reid, N. 1991, AJ, 101, 1304

Idiart, T. P., Maciel, W. J., \& Costa, R. D. D. 2007, A\&A, 472, 101

Jacoby, J. H., \& De Marco, O. 2002, AJ, 123, 269

Kallivayalil, N., van der Marel, R. P., \& Alcock, C. 2006a, ApJ, 652, 121

Kallivayalil, N., van der Marel, R. P., Alcock, C., et al. 2006b, ApJ, 638, 772

Karachentsev, I., Aparicio, A., \& Makarova, L. 1999, A\&A, 352, 363

Kim, M., Kim, E., Lee, M. G. et al. 2002, AJ, 123, 244

King, J. R. 2000, AJ, 120, 1056

Lee, M. G. 1993, ApJ, 408, 409

Lehner, R., Howk, J. C., Keenan, F. P., et al. 2008, ApJ, 678, 219

Leisy, P., \& Dennefled, M. 2006, A\&A, 456, 451

Ma, J., Zhou, X., \& Chen, J. 2004, A\&A, 413, 563

Magrini, L., Corbelli, E., \& Galli, D., 2007, A\&A, 470, 843

Magrini, L., Stanghellini, L., \& Villaver, E. 2009, ApJ, 696, 729

Marigo, P., Girardi, L., \& Bressan, A. 1999, A\&A, 344, 123

Majewski, S. M., Frinchaboy, P. M., Peter, M., et al. 2005, AJ, 130, 2677

Martin, P., \& Roy, J.-R. 1994, ApJ, 424, 599
Mastropietro, C. 2008, The Magellanic System: Stars, Gas, and Galaxies, ed. J.Th. van Loon, \& J.M. Oliveira, IAU Symp., 256, 117

McConnachie, A.W., Alan W., Chapman, S. C., et al., 2006a, ApJ, 647, L25

McConnachie, A. W., Alan, W., Arimoto, N., et al. 2006b, MNRAS, 373, 715

McLean, I. S., \& Liu, T. 1996, ApJ, 456, 499

Minniti, D., \& Zijlstra, A. A. 1997, AJ, 114, 147

Minniti, D., Borissova, J., Rejkuba, M., et al. 2003, Sci., 301, 1508

Momany, Y., Held, E. V., Saviane, I., et al. 2002, A\&A, 384, 393

Nidever, D. L., Majewski, S. R., \& Butler, W. B. 2008, AJ, 679, 432

Nöel, N. E., \& Gallart, C. 2007, AJ, 665, L23

Parisi, M. C., Grocholski, A. J., Geisler, D., et al. 2009, AJ, 138, 517

Paturel, G., Petit, C., Prugniel, P., et al. 2003, A\&A, 412, 45

Piatti, A. E., Sarajedini, A., Geisler, D., et al. 2005, MNRAS, 358, 1215

Pompéia, L., Hill, V., Spite, M., et al. 2008, A\&A, 480, 379

Pont, F., Zinn, R., Gallart, C., et al. 2004, AJ, 127, 840

Reid, W. A., \& Parker, Q. A. 2006, MNRAS, 373, 521

Roškar, R., Debattista, V. P., Stinson, G. S., et al. 2008, ApJ, 675, L65

Rosolowsky, E., \& Simon, J. D. 2008, ApJ, 675, 1213

Sarajedini, A., Geisler, D., Schommer, R., et al. 2000, AJ, 120, 2437

Sarajedini, A., Barker, M. K., Geisler, D., et al. 2006, AJ, 132, 1361

Schommer, R. A., Christian, C. A., Caldwell, N., et al. 1991, AJ, 101, 873

Sellwood, J. A., \& Binney, J. J. 2002, MNRAS, 336, 785

Skillman, E. D., Tolstoy, E., Cole, A. A., et al. 2003, ApJ, 596, 253

Soubiran, C., \& Girard, P. 2005, A\&A, 438, 139

Subramaniam, A. 2006, A\&A, 449, 101

Subramanian, S., \& Subramaniam, A. 2009, A\&A, 496, 399

Tiede, G. P., Sarajedini, A., \& Barker, M. K. 2004, AJ, 128, 224

Tolstoy, E., Venn, K. A., Shetrone, M., et al. 2003, AJ, 125, 707

Tosi, M., Gallagher, J., \& Sabbi, E., et al. 2008, Low-Metallicity Star Formation: From the First Stars to Dwarf Galaxies, ed. L. K. Hunt, S. Madden, \& R. Schneider, IAU Symp., 255, 381

van der Marel, R. P. 2001, AJ, 122, 1827

van der Marel, R. P., \& Cioni, M.-R. L. 2001, AJ 122, 1807

van der Marel, R. P., Alves, D. R., Hardy, E., et al. 2002, AJ, 124, 2639

van der Marel, R. P., Kallivayalil, N., \& Besla, G. 2008, The Magellanic System: Stars, Gas, and Galaxies, ed. J. Th. van Loon, \& J. M. Oliveira, IAU Symp., 256,81

Vlajić, M., Bland-Hawthorn, J., \& Freeman, K. C. 2009, ApJ, 697, 361

Wakker, B. P. 2001, ApJS, 136, 463

Williams, B. F., Dalcanton, J. J., Dolphin, A. E., et al. 2009, ApJ, 695, L15

Zaritsky, D., Kennicutt, R. C., Jr., \& Hucra, J. P. 1994, ApJ, 420, 87

Zaritsky, D., Harris, J., Thompson, I. B., et al. 2002, AJ, 123, 855

Zaritsky, D., Harris, J., Thompson, I. B., et al. 2004, AJ, 128, 1606 